\title{
三维虚拟现实环境中基于EEG的异步BCI小车导航系统
}

\author{
赵启斌 ${ }^{\circledR}, 弓$ 丽清 $\mathbb{1}^{*}$, CICHOCKI Andrzej ${ }^{\circledR}$
}

(1) 上海交通大学计算机科学与工程系, 上海 200240 ;

(2) Laboratory for Advanced Brain Signal Processing, RIKEN Brain Science Institute, Saitama 351-0198, Japan

*联系人, E-mail: zhang-lq@,cs.sjtu.edu.cn

2008-08-08 收稿, 2008-10-24 接受

国家重点基础研究发展计划(批准号: 2005CB724301)和国家高技术研究发展计划(批准号: 2006AA01Z125)资助项目

摘要 脑机交互(BCI)目的是为了建立一种全新的对外信息交流通路, 它不依赖于常 规大脑输出通道(外周神经系统及肌肉组织). 但是, 在大脑与计算机之间建立一种自 然的、功能复杂的交互方式仍是一种挑战. 本文研究了如何使运动想象(MI)引起的事 件相关去同步/同步(ERD/ERS)的持续时间可以被思维任务调制，并提供一种额外的 连续控制参数，从而超越了传统的二值控制问题. 另外，采用持续去同步的非锁时性， 开发了基于累积增量控制策略的异步 BCI 系统, 即三维虚拟现实中小车导航系统. 实 时实验结果表明, 本文所提出的方法能够使受试者采用运动想象 EEG 在复杂的三维 虚拟现实环境中平稳地驾驶小车.
关键词

脑机交互

脑电图

事件相关去同步/同步

公共空间频率模式

任务相关持续去同步/同步
大脑与外部电子设备之间的实时交互可以代替 因受伤或瘫疾而失去的部分感知与运动功能 ${ }^{[1,2]}$. 对 于侵入式脑机交互(brain computer interface, BCI)的 研究表明, 猴子与人类能够利用脑信号来控制外部 设备, 如计算机鼠标等 ${ }^{[3-9]}$. 而非侵入式BCI近年来也 成为神经科学、神经康复工程和信号处理领域的热门 研究课题 ${ }^{[10 \sim 14]}$. BCI系统能够从头皮采集的脑电图 (electroencephalogram, EEG)信号中读出用户的思维 意向, 从而用于基本的通信与控制 ${ }^{[15 \sim 18]}$. 受试者必须 学习自主控制EEG信号特征. 另外, BCI系统需要采 用高级机器学习与信号处理技术来自适应特定受试 者的脑信号 ${ }^{[19 ~ 23]}$. 尽管BCI希望能利用脑信号为残疾 人建立新型的通信通道, 近年来也有研究集中在开 发康复工程中的潜在应用、多媒体通信、虚拟现实以 及电子娱乐等方面.

但通常认为, 复杂的控制功能, 如机器人手臂、 神经假肢等很难用非侵入式EEG来实现，因为EEG信 号反映的是上百万神经元活动的混合结果, 从而空 间分辨率非常低 ${ }^{[26,27]}$. 另外, 非侵入式 $\mathrm{BCI}$ 相对较低 的性能也是研究中的一大困难. 事实上, 大部分现有
基于EEG的BCI系统的速度与准确率还远低于眼动交 互系统，信息传输率通常低于 $0.5 \mathrm{~b} / \mathrm{s}$.

本研究是为了建立一种新型的非侵入式运动想 象 BCI 原型系统，采用从头皮采集的 EEG 信号来实 现更加复杂的实时控制任务(如驾驶汽车), 不仅采用 了运动想象的类别(如想象左、右手、脚)来控制汽车 方向盘的转动方向, 并且利用特定想象任务的持续 时间来定义相应控制命令的强度, 并用来控制连续 变化的方向盘转动角度. 因此用户可以通过调整方 向盘角度来自主驾驶汽车, 并且可以加速或自动减 速. 为了达到该控制能力, 设计了一种累积增量控制 策略, 以增量形式来连续控制当前想象任务持续时 间并实时反映当前想象任务的强度. 相对于传统 BCI 中两种或三种离散控制命令, 本系统具有更加精细 与平滑的控制能力, 如汽车速度与方向盘角度的连 续变化可通过用户调整特定想象任务引起的 $\mathrm{ERD} / \mathrm{ERS}$ 的持续时间来控制. 在此基础上, 该 $\mathrm{BCI}$ 系统采用了灵活的异步操作协议使受试者能够通过 多种不同的运动想象任务来自主连续地控制 $\mathrm{BCI}$ 系 统. 
实时实验结果表明，非侵入式 BCI 系统能够得 到较复杂的控制任务, 可以在快速变化的三维虚拟 现实中驾驶汽车. 并且, 本文提出的 BCI 通过选择最 优的滑动窗口长度 (即信息传输率最高的窗口长度) 来得到较好的系统可靠性与反应速度之间的平衡.

\section{1 任务相关持续去同步}

我们的目的是通过神经生理学现象建立一种新 型的BCI交互机理. 众所周知, 肢体运动与想象都会 引起感觉运动节律(sensorimotor rhythms, SMR)的降 低及其运动结束后的升高, 该现象称为事件相关去 同步(event related desynchronization, ERD)与事件相 关同步 (event related synchronization, ERS $)^{[28,29]}$. 研究 表明, 运动想象引起大脑运动皮层呈现短暂的ERD/ ERS现象, 且该反应与提示信号具有锁时性. 然而, 大部分研究都集中在检测ERD/ERS现象是否出现及 其潜伏期，采用提示信号后特定时间窗口内检测，并 假设单次事件在一定的短时间内产生单个ERD/ERS. 因此, 现有的基于 ERD/ERS的BCI系统通常难以实现 连续以及异步控制.

本文研究受试者是否能够产生连续或持续的可 以被看作稳态运动想象任务的 ERD/ERS 现象，类似 于稳态视觉诱发电位 (steady-state visual evoked potential, SSVEP)或者听觉稳态反应(auditory steadystate responses, ASSR). 采用此概念, 本文设计了另 外一种实验范式, 要求受试者以近似固定的频率连 续并重复地想象任务, 如手握动作. 实验结果证实了 该假设, 当想象任务连续并重复执行时, ERD 现象也 会持续出现. 该现象理论上可以解释为重复的运动 想象任务引起了皮层反应进入一种“稳态”，并且在 大脑皮层相应的运动控制区产生持续的去同步现象.

通过对 4 名受试者(24 30 岁男性)进行实验, 结 果有 3 名受试者能够产生持续去同步现象. 图 1 中 EEG 时频图证明了该假设, 并可明显看到 ERD/ERS 现象在整个 $4 \mathrm{~s}$ 过程中持续存在. 该现象可解释为: 当持续想象左/右手运动时, 对侧区域会在整个想象 任务中处于激活状态，而同侧区域处于非激活状态， 因而导致了持续的对侧 ERD 与同侧 ERS, 并称之为 任务相关持续去同步/同步(task related sustained desynchronization and synchronization, TRSD/TRSS) 或稳态事件相关去同步/同步. TRSD/TRSS 是由固定 频率的多次运动想象任务而产生，可以被视作特定
事件连续单次 ERD/ERS 的重叠组合.

总之, $\mathrm{ERD} / \mathrm{ERS}$ 是由离散短暂事件引起, 而 TRSD/TRSS 是由连续重复的任务引起. TRSD/TRSS 主要特点是能够同时提供关于想象任务的持续时间 信息, 为 $\mathrm{BCI}$ 提供了一种额外的控制参数, 且该参数 为连续量. 相对于现有的基于 MI 的 BCI, TRSD 的持 续时间可以被定义为特定想象任务的强度参数, 因 而可以提供更加复杂的控制功能. 并且, ERD/ERS 与 提示信号具有非锁相性, 但与提示信号具有锁时性, 然而 TRSD/TRSS 与提示信号不仅具有非锁相性且具 有非锁时性, 从而不同类型的 MI 之间的转换可以通 过 TRSD 模式检测来连续识别, 所以更加适合于异步 $\mathrm{BCI}$ 系统.

\section{2 三维虚拟现实中脑驱车}

我们开发了一种新型异步 $\mathrm{BCI}$ 系统, 也称作“脑 驱车”系统, 其任务是在无任何外部同步信号刺激下, 在三维虚拟现实中通过运动想象 EEG 来驾驶虚拟汽 车. 为了测试受试者是否能够自由控制虚拟汽车的 方向与速度, 并沿着虚拟现实环境中公路行驶, 设计 了两个虚拟场景, 分别采用不同的公路设计来评价 受试者 BCI 性能.

场景 I：笔直公路中心线上放置许多雉形交通 障碍, 且等距离摆放. 任务是驾驶汽车绕过每一个交 通障碍，并沿着 $\mathrm{S}$ 型路线行驶，如图 2(a)所示.

场景 II: 闭合的曲线公路系统, 并带有多个左/ 右急转弯, 如图 2(b)所示. 任务是驾驶汽车沿着公路 尽可能以最快速度行驶.

对于上述两种场景的评价准则基本相似, 受试 者驾驶的汽车如果触碰公路边界即为失败. 在完成 任务的前提下，尽可能以较快的速度驾驶汽车.

为了翻译思维相关的 EEG 信号为输出命令并控 制虚拟汽车在虚拟现实环境中平稳行驶, $\mathrm{BCI}$ 系统的 输出命令用来控制汽车方向盘与加速器, 而不是直 接控制汽车的方向和位置. 在场景中的每一个转弯, 方向盘的转动角度必须准确并且及时, 否则都有可 能驶出公路边界. 但由于 EEG 信号的高度非稳态性 以及错误分类现象的不可避免性, 从而使得驾驶非 常困难. 因此, 相对于离散控制命令的 $\mathrm{BCI}$ 系统, 本 系统最大优点在于能够提供准确、鲁棒的角度控制, 并最大限度的降低因错误分类引起的影响.

为了成功实现该驾驶策略, 系统需能够识别超 


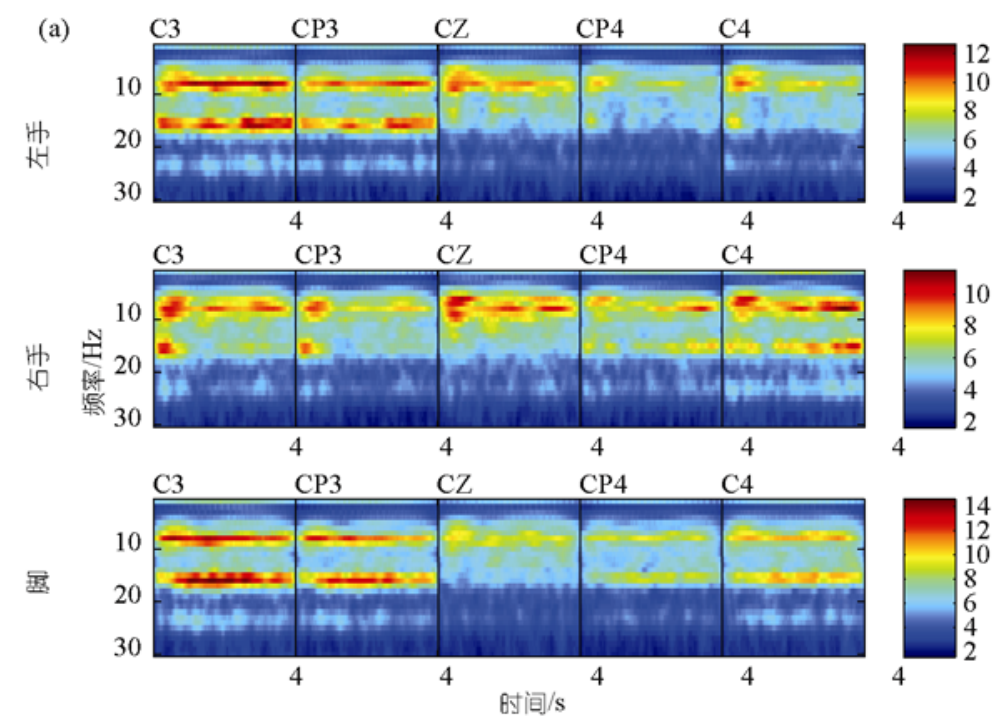

(b)

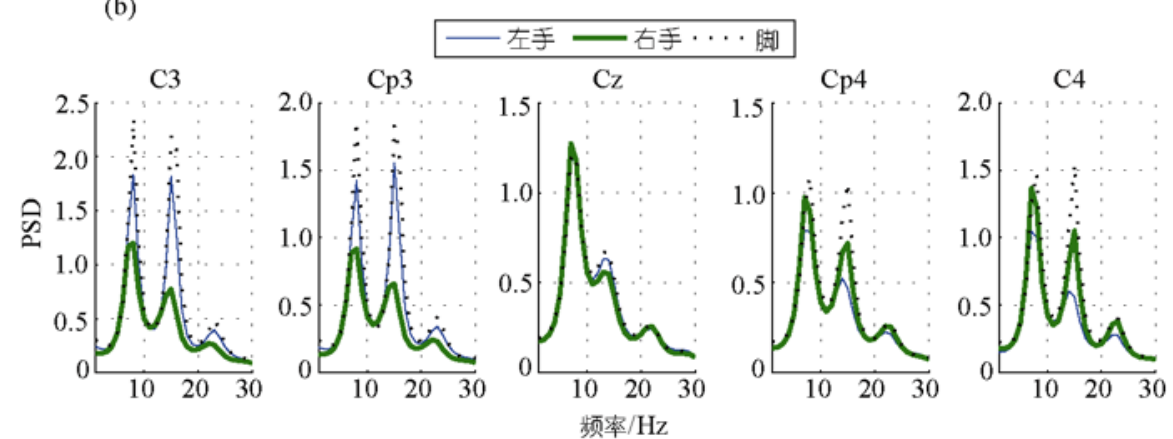

图 1 TRSD/TRSS 现象

(a) 3 种运动想象任务(左手、右手、脚)过程中空间 5 导 EEG 的平均时频分布. 第 1 行中明显可见当左手想象任务时, C3, Cp 3 上出现 TRSS 以及 $\mathrm{C} 4, \mathrm{Cp} 4$ 上出现 TRSD 现象. 类似地, 第 2 行中可见当右手想象任务时相反的区域出现 TRSD/TRSS 现象. 第 3 行中当脚想象运动 时, $\mathrm{Cz}$ 上的 TRSD 明显, C4, Cp4 上也呈现轻微的 TRSD 现象. (b)空间 5 导的平均能量谱分布说明了不同的运动想象任务在空间上能量 分布的区别

过 3 种 MI 状态(左手、右手以及脚). 值得注意的是, 本研究采用后验概率来解决 BCI 中“休息”状态的检 测, 即受试者无任何想象动作. 当分类器对各类任务 的后验概率均小于一特定阈值时(通常 $90 \%$ ), 便视为 休息状态. 因此, 共有 4 种状态 (左手、右手、脚、休 息)被解码并分别投射到 4 种控制命令, 即转左(L)、 转右 $(\mathrm{R})$ 、加速 $(\mathrm{S})$ 以及无命令 $(\mathrm{NC})$.

实时 BCI过程中, 汽车的位置与车身方向每 $0.05 \mathrm{~s}$ 根据当前方向盘与加速器状态计算一次并更新虚拟 现实场景. 同时方向盘与汽车尾灯(代表加速或减速) 作为系统的连续反馈呈现给受试者, 如图 2(c)所示. 该反馈信号表示了当前汽车运行状态与当前系统输 出命令的综合结果. 为了能快速检测 MI 状态转换, 采用预定义的滑动窗口对当前窗口 EEG 进行特征提 取与分类, 滑动步长为 $0.125 \mathrm{~s}$. 根据此策略, 受试者
可以自主控制想象任务，如图 2(d)所示.

\section{3 方法}

\section{1 公共空间频率模式}

公共空间模式(common spatial patterns, CSP)算 法是目前通过计算空间滤波来进行特征提取的最有 效算法 ${ }^{[30 \sim 32]}$ 之一. 但当非辨别脑节律信号与最有效 的辨别脑节律信号在频率范围上重叠时，性能会急 剧下降. 另一方面, 用于CSP算法的频带范围需要人 为选择或者定义为较大范围, 这样容易造成因频带 选择不佳引起的性能下降. 本文提出了一种新型算 法即公共空间频率模式(common spatial frequency patterns, CSFP), 能够同时优化空间与频率模式，从 而提高了仅采用少数空间导联的EEG信号的分类准 确率. 总体平均性能上超过CSP, 特别是当最大辨别 

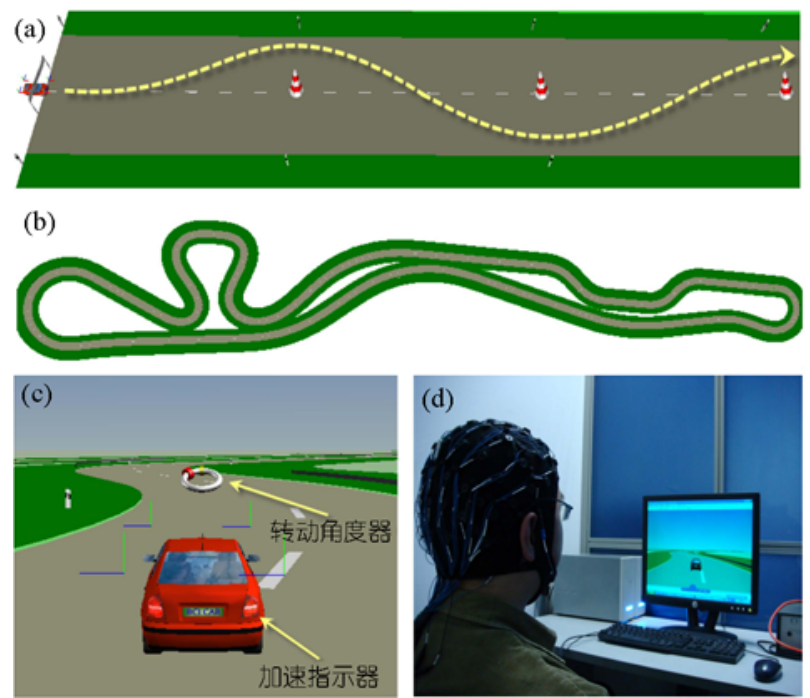

图 2 实验设计

(a) 场景 1 中的笔直公路. 要求受试者驾驶汽车沿着 $S$ 型路线行驶 并避开交通障碍，如图中的黄线所示. (b) 场景 2 中的曲线公路. (c)虚拟现实环境与虚拟小车. 箭头所示为系统反馈信号, 方向盘 指示了转动角度而汽车尾灯指示当前加速器的状态(加速/减速).

(d) 某受试者实时实验过程

节律频率范围与任务类别相关时, 优势更加明显.

为了适应非平稳频率分析, 连续小波变换以不 同频带上可变的窗口大小得到了时域与频域分辨率 之间更好的平衡. EEG 信号 $x^{c, k}(\tau)$ 的连续小波变换 (continuous wavelet transform, CWT) 定义为:

$$
W^{c, k}(a, t)=\frac{1}{\sqrt{a}} \int_{-\infty}^{+\infty} x^{c, k}(\tau) \psi\left(\frac{\tau-t}{a}\right) \mathrm{d} \tau,
$$

其中, $t$ 表示时间平移, $a$ 表示尺度参数, $\psi$ 代表小波函 数, $W^{c, k}(a, t)$ 表示数据段 $x^{c, k}(\tau)$ 的 CWT 变换, $c$ 代表导 联, $k$ 代表单次 EEG. 尽管现有小波函数很多, Morlet 小波更适合于 EEG 信号的时频分析. 由于尺度参数 $a$ 与频率 $f$ 之间关系为 $a=\omega_{0} /(2 \pi f)$, 那么可得到小波时 频分析函数:

$$
\hat{W}^{c, k}(f, t)=W^{c, k}\left(\frac{F_{c}}{f T}, t\right),
$$

其中, $\hat{W}^{c, k}(f, t)$ 表示第 $k$ 次 EEG 在导联 $c$, 频率 $f$, 时 间 $t$ 的小波时频系数. $F_{c}$ 是小波函数中心频率, $T$ 是信 号采样周期. 可通过设置一个频率 $f$ 范围来建立时频 分布(time-frequency distributions, TFD)矩阵, 并定义 为

$$
\boldsymbol{p}_{k}^{c, f}=\hat{W}^{c, k}(f, t) .
$$

因此，可以建立对应每个导联的时频分布矩阵以及
对应每个频率的时空分布矩阵，如下:

$$
\boldsymbol{U}_{k}^{c}=\left(\boldsymbol{p}_{k}^{c, f_{1}} \boldsymbol{p}_{k}^{c, f_{2}}, \ldots, \boldsymbol{p}_{k}^{c, f_{m}}\right)^{T}
$$

和

$$
\boldsymbol{V}_{n}^{f}=\left(\boldsymbol{p}_{k}^{c_{1}, f} \boldsymbol{p}_{k}^{c_{2}, f}, \ldots, \boldsymbol{p}_{k}^{c_{n}, f}\right)^{T},
$$

其中, $\boldsymbol{U}_{k}^{c}$ 表示第 $k$ 次 EEG 在导联 $c$ 上的时频分布, 频 率变化范围是从 $f_{1}$ 到 $f_{m}, \boldsymbol{V}_{k}^{f}$ 表示第 $k$ 次 EEG 在频率 $f$ 上的时空分布, 空间变化范围是从 $c_{1}$ 到 $c_{n}$. 为了重 组单次 EEG 信号时频分布, 把所有导联上的 $\boldsymbol{U}_{k}^{c}$ 或所 有频率上的 $\boldsymbol{V}_{k}^{c}$ 叠放在一起，便可得到

$$
\boldsymbol{Y}_{k}=\left(\begin{array}{c}
\boldsymbol{U}_{k}^{c_{1}} \\
\boldsymbol{U}_{k}^{c_{2}} \\
\vdots \\
\boldsymbol{U}_{k}^{c_{n}}
\end{array}\right) \text { 或 } \boldsymbol{Y}_{k}=\left(\begin{array}{c}
\boldsymbol{V}_{k}^{f_{1}} \\
\boldsymbol{V}_{k}^{f_{2}} \\
\vdots \\
\boldsymbol{V}_{k}^{f_{m}}
\end{array}\right) .
$$

在此基础上，定义类协方差矩阵为:

$$
\boldsymbol{\Gamma}_{i}=\sum_{k \in \text { class }_{i}} \frac{\boldsymbol{Y}_{k} \boldsymbol{Y}_{k}^{T}}{\operatorname{tr}\left(\boldsymbol{Y}_{k} \boldsymbol{Y}_{k}^{T}\right)},
$$

其中, $\boldsymbol{Y}_{k} \in R^{N \times M}$ 表示第 $k$ 次 EEG 的时频分布, $N$ 等于 导联数 $\times$ 频率数(即 $n \times m), M$ 表示单次 EEG 采样数, $\operatorname{class}_{i}$ 表示属于第 $i$ 类的训练数据.

CSFP 算法的优化目标是为了找到最大辨别的空 间和频率复合模式，通常用 $\boldsymbol{W}$ 来表示，使得向量 $\boldsymbol{w}_{p}$ $\in R^{d}(d=n \times m)$, 也就是 $\boldsymbol{W}$ 中第 $p$ 列, 能够最大化某 一类的方差并同时最小化各类总协方差. 其中每一 个 $\boldsymbol{w}$ 的计算可通过优化如下的目标函数:

$$
\underset{w}{\operatorname{argmax}}=\frac{\boldsymbol{w}^{T} \boldsymbol{S}_{I} \boldsymbol{w}}{\boldsymbol{w}^{T} \boldsymbol{S}_{T} \boldsymbol{w}} .
$$

为了得到每一类的最大辨别模式, 可设置 $\boldsymbol{S}_{I}$ 和 $\boldsymbol{S}_{T}$ 为:

$$
\boldsymbol{S}_{I}=\left.\boldsymbol{\Gamma}_{i}\right|_{i=1} ^{M}, \quad \boldsymbol{S}_{T}=\frac{1}{M} \sum_{i=1}^{M}\left(\boldsymbol{\Gamma}_{i}\right),
$$

其中 $S_{I}$ 表示对应于第 $i$ 类 TFD 的协方差矩阵，见式(7); $\boldsymbol{S}_{T}$ 表示所有 $M$ 类数据的总协方差矩阵. 根据式(8)中 的优化目标, 可得到对应于每类 $i$ 的投影矩阵 $\boldsymbol{W}^{(\mathrm{i})}$, 也就是空间频率模式. $\boldsymbol{W}^{(\mathrm{i})}$ 中的投影向量数可通过交 叉验证来选择最优数目. 最后, 把所有投影矩阵 $\left.\boldsymbol{W}^{(i)}\right|_{i=1} ^{M}$ 组合在一起得到 $\boldsymbol{W}_{\mathrm{csfp}}=\left[\boldsymbol{W}^{(1)}, \boldsymbol{W}^{(2)}, \cdots, \boldsymbol{W}^{(M)}\right]$, 可定义为多类 EEG 信号的最大辨别空间频率模式.

通过优化得到投影矩阵后, EEG 时频分布矩阵 $\boldsymbol{Y}_{k}$ 可以投影到 $\boldsymbol{W}_{\mathrm{csfp}}$ 得到:

$$
\boldsymbol{Z}_{k}=\boldsymbol{W}_{\mathrm{csfp}}^{T} \boldsymbol{Y}_{k} .
$$


因此, $\boldsymbol{Z}_{k}$ 表示多类数据最大辨别分量, 该分量通过同 时最优化空间与频率模式而得到.

最终, 根据式(10)对 EEG 数据的 TFD 进行投影 得到用来分类的特征向量. 通常, $M$ 类中的各类只需 要保留较少数(如 $l$ ) 个的投影分量, 其中包含了该类 最大辨别信息. 投影分量的选择是根据 $\boldsymbol{W}_{\mathrm{csfp}}$ 中 $l$ 个最 大特征值对应的投影向量来选择. 得到投影分量 $\boldsymbol{Z}_{k}^{p}, p=1, \cdots, M \times l$ 后, 为了得到分类器训练时输入的 特征向量, 需对投影后的分量方差作标准化与 $\log$ 变 换处理，

$$
\boldsymbol{f}_{k}=\log \left(\frac{\operatorname{diag}\left(\boldsymbol{Z}_{k} \boldsymbol{Z}_{k}^{T}\right)}{\operatorname{tr}\left(\boldsymbol{Z}_{k} \boldsymbol{Z}_{k}^{T}\right)}\right),
$$

其中, $\boldsymbol{f}_{k} \in R^{M \times l}$ 是第 $k$ 次 EEG 投影后的 CSFP 特征向 量, $\log$ 变换目的是使数据近似平均分布.

但由于特征向量 $\boldsymbol{f}_{k}$ 仅包含了各 CSFP 分量之间相 对的能量分布信息, 且实验表明所有 CSFP 分量的总 能量对想象任务也具有重要的辨别能力, 特别是脚 想象与左右手想象之间的区别更为明显. 为了进一 步提高分类性能，可定义另外一部分特征向量如下:

$$
\boldsymbol{d}_{k}^{j}=\mid \sum_{i=1}^{M \times l} \operatorname{var}\left(\boldsymbol{Z}_{k}^{i}\right)-\frac{1}{N_{j}} \sum_{k \in \text { class }_{j}}\left(\sum_{i=1}^{M \times l} \operatorname{var}\left(\boldsymbol{Z}_{k}^{i}\right)\right),
$$

其中, $j$ 表示类别, $1 \sim M$ 之间变化, $N_{j}$ 是属于第 $j$ 类的单 次 EEG 数目, 而 $\boldsymbol{d}_{k}^{j}$ 表示所有 CSFP 分量总能量与 $j$ 类平均的 CSFP 分量总能量之间的距离. 通过单位化 操作 $\boldsymbol{d}_{k}^{j} /\left\|\boldsymbol{d}_{k}^{j}\right\|^{2}$, 可得到 $k$ 次 EEG 的特征向量, 并反 映了与各类之间距离分布情况. 通过特征向量组 合 $\left[\boldsymbol{f}^{T}, \boldsymbol{d}^{T}\right]^{T}$, 得到最终的用来训练分类器的特征向 量. 分类器采用线性支持向量机 (support vector machine, SVM), 并使用 $10 \times 10$ 交叉验证来训练分类 器, 得到最优参数及模型.

\section{2 累积增量控制策略}

值得注意的是，若仅通过 4 个离散控制命令并不 能得到方向盘角度的精细控制以及速度控制. 因而, 我们使用了特定想象任务的持续时间作为附加的控 制参数, 这样不仅能得到更高级复杂的控制功能, 而 且能够防止因错误分类而产生的汽车方向的快速左 右振动现象.

对于方向盘角度的控制, 由左右手运动想象的 分类决定旋转方向, 并采用特定 TRSD 的持续时间即 相应的想象任务的持续时间来决定具体的转动角度
值. 持续时间越长, 产生的转动角度也越大, 这样使 $\mathrm{BCI}$ 输出命令由离散值转化为连续量. 方向盘角度的 控制是通过当前控制命令持续时间的线性方程来计 算, 定义如下:

$$
\theta_{t}=\left(D_{t}^{L}-D_{t}^{R}\right) \Delta \theta,
$$

其中 $D_{t}^{L}$ 和 $D_{t}^{R}$ 代表左转与右转命令在 $t$ 时刻的持续 时间，即当前时刻之前与当前控制命令完全一致的 时间长度. $\Delta \theta$ 是固定的角速度, 该参数可通过对特定 受试者训练过程中来选择. $\theta_{t}$ 是 $t$ 时刻方向盘的角度 值，其中正值与负值分别表示向左或向右旋转.

对于速度的控制，当检测到脚运动想象对应的 TRSD 状态后, 便打开加速器并以一定的加速度来提 高汽车速度, 相反当脚运动想象状态消失, 则关闭加 速器并以一定的减速度来降低速度. $t$ 时刻速度 $v_{t}$ 是 由 $t-1$ 时刻的速度 $v_{t-1}$ 以及一固定的加速或减速值求 和而得, 迭代式如下:

$$
v_{t}=v_{t-1}+\delta_{t}^{S}\left(\Delta v_{+}+\Delta v_{-}\right)-\Delta v_{-},
$$

其中, $\delta_{t}^{S}$ 是开关函数, 当输出命令为加速 $(\mathrm{S})$ 时等于 1, 否则等于 $0 . \Delta v_{+}$表示固定的加速度, 而 $\Delta v_{-}$表示固 定的减速度, 通常满足 $\Delta v_{+}>\Delta v_{-}$. 此控制机制可以解 释为实时过程中, 一旦检测到 $\mathrm{S}$ 命令后, 汽车速度便 增加 $\Delta v_{+}$一次, 其余情况下汽车速度减少 $\Delta v_{-}$一次, 该控制机制与真实汽车的加速踏板非常相似.

上述控制策略采用了特定运动想象任务的持续 时间作为额外控制参数, 并提供了更复杂的控制能 力, 即“时间换取复杂性”. 并且, NC 状态使汽车方向 盘归零并关闭加速器, 使汽车以减速状态向前行驶. 根据上述策略, 错误分类的结果往往持续时间不长, 因而不会对方向引起很大的影响, 从而使系统具有 了一定的容错能力. 因此, 本文中 BCI 系统相对于现 有大多数 BCI 系统更具有鲁棒性.

为了在线检测特定想象任务的持续时间，本文开 发了一种基于滑动窗口的累积增量控制(cumulative incremental control, CIC)策略. 在每一时刻，特定 MI 的持续时间可以根据当前与历史产生的命令来在线 更新计算. 这样实际输出命令的幅值通过在线方式 累积而得, 方向盘的每次旋转根据是否检测到连续 一致的状态, 并以固定值增量形式来实现. 若当前检 测到的命令与历史命令不一致，则当前控制命令的 幅值设置为 0 , 并且方向盘归 0 . 换句话说, 为了大幅 度地旋转方向盘, 受试者需要持续左/右手 MI 任务较 长的时间; 类似地为了快速地增加速度, 受试者需要 
持续脚 MI 任务较长的时间. 最终使得方向盘角度 $\theta_{t}$ 在 $[-\pi / 2, \pi / 2]$ 范围内连续变化, 而速度值在 $\left[v_{\min }, v_{\max }\right]$ 范围内连续变化. 本文的控制策略是一种自然的控 制方式, 而且非常类似于现实中使用肢体来驾驶真 实汽车过程.

\section{3 虚拟现实环境控制}

在三维虚拟现实环境中的汽车位置与方向每 $0.05 \mathrm{~s}$ 更新一次, 如下式:

$$
\boldsymbol{o}_{t}=\boldsymbol{R}_{c \theta_{t}} \boldsymbol{o}_{t-1} \text { 和 } \boldsymbol{p}_{t}=\boldsymbol{p}_{t-1}+\boldsymbol{o}_{t} v_{t},
$$

其中 $\boldsymbol{o}_{t}$ 表示 $t$ 时刻的三维方向向量, 为单位长度. $\boldsymbol{R}_{c \theta_{t}}$ 表示对应于角度 $c \theta_{t}$ 的旋转矩阵, 其中 $c$ 表示方向 盘与汽车转动角度之间的固定比例参数. 式(15)中可 看出, 当前方向与由前一时刻方向以及式(13)中当前 方向盘的旋转角度 $\theta_{t}$ 相关. $\boldsymbol{p}_{t}$ 代表在虚拟现实环境中 $t$ 时刻的三维位置向量, 与前一时刻位置 $\boldsymbol{p}_{t-1}$, 当前方 向向量 $\boldsymbol{o}_{t}$ 以及式(14)中当前速度 $v_{t}$ 相关.

\section{4 在线实验结果}

4 名健康的男性受试者(年龄: 24 30 岁)参加了本 次实验. 受试者均自愿参加, 并已详细了解了实验过 程、方法及其所可能产生的危险与利益. EEG 信号采 集仅采用 5 导, 即 $(\mathrm{C} 3, \mathrm{Cp} 3, \mathrm{Cz}, \mathrm{Cp} 4, \mathrm{C} 4)$, 分布在运 动感觉区域. 采样率为 $250 \mathrm{~Hz}$.

训练阶段主要包括两部分来提高系统性能: 系 统训练与受试者自适应训练. 每次训练过程包含 30 次任务并持续 3 5 min. 为了能够对运动想象状态连 续分类并检测多种想象任务之间的转换, 训练样本 采用重叠滑动窗口技术从单次任务 EEG 数据段中提 取出多个 EEG 数据段, 滑动窗口间隔为 $0.125 \mathrm{~s}$. 另 外滑动窗口的长度选择至关重要, 直接影响到系统 准确率与反应速度之间的平衡. 本文对实验数据 EEG 分别用不同长度的滑动窗口来分析, 长度变化 0.5 4 s. 当滑动窗口变长时, 分类准确率明显提高, 但导致了 BCI 系统的低反应速度. 因此在训练阶段 中, 根据在线分类性能在 $75 \%$ 以上(安全驾驶汽车最 低要求), 并具有最高信息传输率来选择针对特定受 试者的最优窗口长度.

训练过程中, 由于系统模型与受试者大脑之间 的互相学习与自适应调整, 每位受试者的性能均呈
现逐步提高的现象. 实验结果表明, 3 名受试者在经 过多次训练后, 准确率能够达到 75\%以上, 而另外一 名受试者准确率仅为 $70 \%$, 继续训练后无明显提高. 其中两名受试者 $(\mathrm{S} 1, \mathrm{~S} 2)$ 的性能最优, 且多次运行中 性能较为稳定. 这两名受试者的在线分类性能对于 4 $\mathrm{s}$ 的想象任务能达到 $91 \%$, 甚至对于 $1 \mathrm{~s}$ 的想象任务 也能达到 $76 \%$. 当采用 $1 \mathrm{~s}$ 窗口时, 受试者 S2 的平均 信息传输率可达到 $0.55 \mathrm{bps}$, 特别是在 $0.5 \mathrm{~s}$ 想象任务 时间下, 最高准确率可达到 $70 \%$, 因此信息传输率接 近 $0.8 \mathrm{bps}$. 当想象时间小于 $0.5 \mathrm{~s}$ 时, 分类性能会急 剧下降. 在受试者自适应训练中, 我们发现受试者 $\mathrm{S} 1, \mathrm{~S} 2$ 能够较快地改变输出命令的状态, 最快情况下 甚至在 $0.5 \mathrm{~s}$ 内; 而对于特定想象任务最长的持续时 间为 $4 \sim 6 \mathrm{~s}$.

在虚拟现实环境驾驶汽车过程中, 受试者 $\mathrm{S} 1, \mathrm{~S} 2$ 能够较成功地在两种场景下完成任务并沿着特定路 线行驶. 为了进一步说明在线实验结果, 图 3(a)表示 了在场景 1 中某次运行的连续输出命令序列. 实时的 速度变化与方向盘角度变化分别如图 3(b)和(c)所示, 从中可说明速度与角度的控制是由运动想象的类型 与持续时间共同来控制. 执行特定想象任务的持续 时间越长, 方向盘的转动角度也就越大. 另外, 图 3(c)中可明显看到部分错误分类情况, 但由于相对于 正确分类情况, 持续时间较短, 因此仅造成了较小的 角度偏离, 从而汽车的行驶轨迹几乎不会受错误分 类影响. 实时的汽车行驶路径如图 3(d)所示, 该实验 结果进一步证明了 BCI 系统的鲁棒性.

不同受试者之间更高级的评价准则是比较特定 时间间隔内驾驶汽车行驶的距离. 行驶距离长则说 明该受试者不仅能够正确控制方向盘角度并通过交 通障碍, 而且能够以较快的速度驾驶汽车. 本文实验 中, 受试者 $\mathrm{S} 1, \mathrm{~S} 2$ 分别在 $2 \mathrm{~min}$ 内通过了 7 个和 10 个交通障碍. 为了进一步说明实时实验过程, 我们提 供了两个短的实时实验过程视频 ${ }^{1}$.

另外, 非常有趣的是当受试者 S2 同时执行左手 与左脚(LHF)或右手与右脚(RHF)想象运动时, 大脑 对侧 TRSD 与同侧 TRSS 现象更加明显. 因此得到了 比采用三种基本想象任务(左手、右手和脚)更好的分 类性能. 该现象可解释为: 左/右脚想象运动因为更多 数量的神经元处于了激活状态, 因而同时引发了中心

1) URL: http://bcmi.sjtu.edu.cn/ zhaoqibin/demos.html 

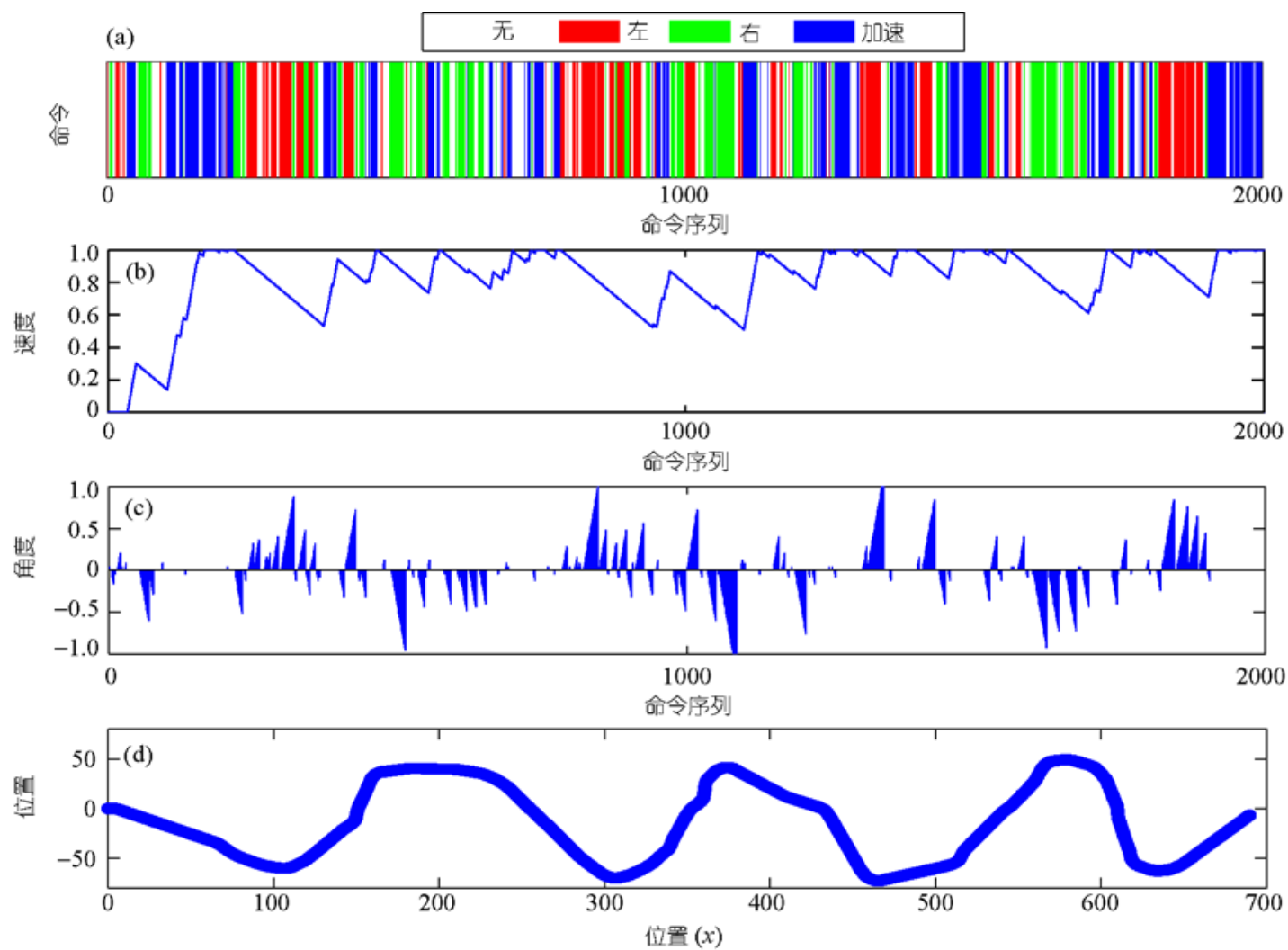

(e)
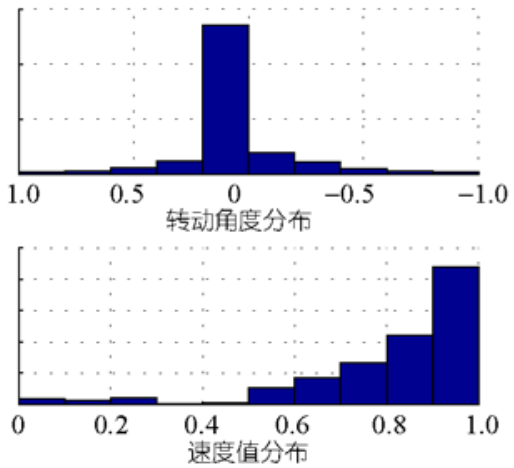

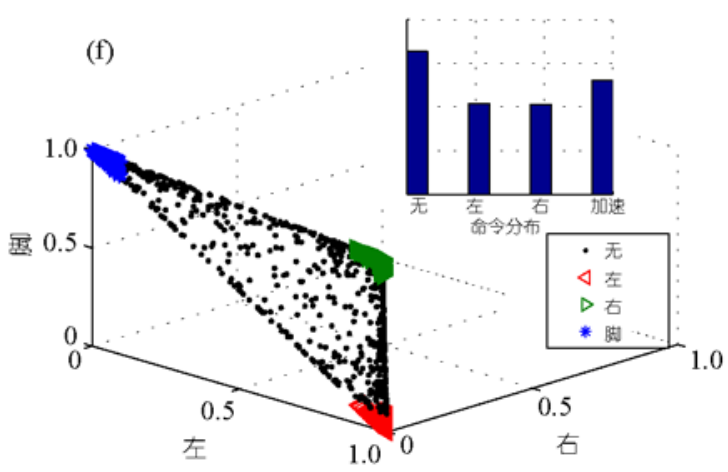

图 3 实时实验结果

(a) 驾驶汽车过程中的命令序列, 即实时对运动想象任务连续分类结果. 左手 $(\mathrm{L}) 、$ 右手 $(\mathrm{R}) 、$ 脚 $(\mathrm{F})$ 想象任务分别影射到左、右和加速命 令, 并加入无命令 $(\mathrm{NC})$ 状态检测. (b) 汽车速度的实时变化过程. 每个时间点表示当前的汽车速度值并与(a)中命令序列一一对应. (c) 对 应于命令序列的实时方向盘角度变化. 角度变化范围是 $[-\pi / 2, \pi / 2]$, 其中正值表示往左旋转, 负值表示往右旋转. 较大的角度值是由较 长的想象持续时间而产生. (d) 汽车在三维虚拟现实中实际行驶路线. (e) 方向盘角度与汽车速度值的出现概率分布. (f) 对应于 3 类想象 任务的后验概率分布. 图中每个点代表命令序列中每次分类的 3 类后验概率. 输出命令为后验概率达到一定的阈值 (红、绿、蓝区域)的 类别, 若 3 类概率均未达到阈值, 则输出无命令状态(黑色区域)

与对侧皮层的 ERD 现象. 因此手与脚的同时想象(如 LHF, RHF)能够产生对侧区域更强的 TRSD 现象.

\section{5 总结}

总而言之，本文研究并开发了一种新型的基于 运动想象的 $\mathrm{BCI}$ 系统, 并有希望控制更多的外部设
备，如残疾人轮椅或用来控制新型的娱乐游戏. 毫无 疑问，对于 $\mathrm{BCI}$ 的现实应用，还需要解决许多基本的 技术困难. 本文中提出的 TRSD/TRSS 现象以及脑驱 车系统促进了异步 BCI 系统的研究. 可以预测在将 来的某一天, 瘫疾病人有可能通过大脑思维活动来 驾驶真实汽车. 


\section{参考文献}

1 Nicolelis M A L. Actions from thoughts. Nature, 2001, 409(6818): 403-407 [doi]

2 Wolpaw J R, Birbaumer N, McFarland D J, et al. Brain-computer interfaces for communication and control. Clin Neurophysiol, 2002, 113(6): 767-791[doi]

3 Dornhege G. Toward Brain-Computer Interfacing. Cambridge, MA: MIT Press, 2007

4 Serruya M, Hatsopoulos N, Paninski L, et al. Instant neural control of a movement signal. Nature, 2002, 416(6877): 141-142[doi]

5 Wessberg J, Stambaugh C R, Kralik J D, et al. Real-time prediction of hand trajectory by ensembles of cortical neurons in primates. Nature, 2000, 408(6810): 361-365 [doi]

6 Taylor D M, Tillery S I H, Schwartz A B. Direct cortical control of 3D neuroprosthetic devices. Science, 2002, 296(5574): 1829 [doi]

7 Musallam S, Corneil B D, Greger B, et al. Cognitive control signals for neural prosthetics. Science, 2004, 305(5681): 258-262 [doi]

8 Santhanam G, Ryu S I, Yu B M, et al. A high-performance brain-computer interface. Nature, 2006, 442(7099): 195-198 [doi]

9 Chapin J K, Moxon K A, Markowitz R S, et al. Real-time control of a robot arm using simultaneously recorded neurons in the motor cortex. Nat Neurosci, 1999, 2: 664-670 [doi]

10 Müller K R, Blankertz B. Toward noninvasive brain-computer interfaces. IEEE Signal Processing Magazine, 2006, 23(5): 125-128

11 Pfurtscheller G, Brunner C, Schlögl A, et al. Mu rhythm (de) synchronization and EEG single-trial classification of different motor imagery tasks. NeuroImage, 2006, 31(1): 153-159 [doi]

12 Blankertz B, Dornhege G, Krauledat M, et al. The non-invasive berlin brain-computer interface: Fast acquisition of effective performance in untrained subjects. NeuroImage, 2007, 37(2): 539-550 [doi]

13 Blankertz B, Tomioka R, Lemm S, et al. Optimizing spatial filters for robust EEG single-trial analysis. IEEE Signal Processing Magazine, 2008, 25(1): 41-56 $\underline{\text { [doi] }}$

14 Sitaram R, Zhang H, Guan C, et al. Temporal classification of multichannel near-infrared spectroscopy signals of motor imagery for developing a brain - Computer interface. NeuroImage, 2007, 34(4): 1416-1427[doi]

Birbaumer N, Ghanayim N, Hinterberger T, et al. A spelling device for the paralysed. Nature, 1999, 398(6725): 297 -298 [doi]

Pfurtscheller G, Neuper C, Muller G R, et al. Graz-BCI: State of the art and clinical applications. IEEE Transactions on Neural Systems and Rehabilitation Engineering, 2003, 11(2): 1-4

17 Wolpaw J R, McFarland D J, Bizzi E. Control of a Two-dimensional movement signal by a noninvasive brain-computer interface in humans. Proc Natl Acad Sci USA, 2004, 101(51): 17849-17854 [doi]

18 Wolpaw J R, McFarland D J, Vaughan T M, et al. The Wadsworth center brain-computer interface (BCI) research and development program. IEEE Transact Neur Syst Rehabilit Eng, 2003, 11(2): 1-4[doi]

19 Muller K R, Krauledat M, Dornhege G, et al. Machine learning techniques for brain-computer interfaces. Biomed Tech, 2004, 49(1): $11-22$

20 Dornhege G, Blankertz B, Krauledat M, et al. Combined optimization of spatial and temporal filters for improving brain-computer interfacing. IEEE Trans Biomed Eng, 2006, 53(11): 2274-2281 [doi]

21 Blankertz B, Dornhege G, Lemm S, et al. The berlin brain-computer interface: Machine learning based detection of user specific brain states. J Univ Comp Sci, 2006, 12(6): 581-607

22 Muller K R, Anderson C W, Birch G E. Linear and nonlinear methods for brain-computer interfaces. IEEE Transact Neur Syst Rehabilit Eng, 2003, 11(2): 165-169 [doi]

23 Kamousi B, Liu Z, He B. Classification of motor imagery tasks for brain-computer interface applications by means of two equivalent dipoles analysis. IEEE Transact Neur Syst Rehabilit Eng, 2005, 13(2): 166-171 [doi]

Pfurtscheller G, Leeb R, Keinrath C, et al. Walking from thought. Brain Res, 2006, 1071(1): 145-152 [doi]

Krepki R, Blankertz B, Curio G, et al. The berlin brain-computer interface (BBCI)-towards a new communication channel for online control in gaming applications. Multimedia Tools Appl, 2007, 33(1): 73-90 [doi]

26 Fetz E. Real-time control of a robotic arm by neuronal ensembles. Nat Neurosci, 1999, 2: 583 - 584 [doi]

27 Donoghue J. Connecting cortex to machines: Recent advances in brain interfaces. Nat Neurosci, 2002, 5(suppl): 1085 -1088 [doi]

28 Pfurtscheller G, Lopes da Silva F. Event-related EEG/MEG synchronization and desynchronization: basic principles. Clin Neurophysiol, 1999, 110(11): 1842-1857 [doi]

29 Pfurtscheller G, Neuper C, Brunner C, et al. Beta rebound after different types of motor imagery in man. Neurosci Lett, 2005, 378(3): $156-159$ [doi]

30 Ramoser H, Muller-Gerking J, Pfurtscheller G. Optimal spatial filtering of single trial EEG during imagined handmovement. IEEE Transact Neur Syst Rehabilit Eng, 2000, 8(4): 441-446 [doi]

31 Müller-Gerking J, Pfurtscheller G, Flyvbjerg H. Designing optimal spatial filters for single-trial EEG classification in a movement task. Clin Neurophysiol, 1999, 110: 787-798 [doi]

32 Wang Y, Zhang Z, Li Y, et al. BCI competition 2003-data set IV: An algorithm based on CSSD and FDA for classifying single-trial EEG. IEEE Transact Biomed Eng, 2004, 51(6): 1081-1086 [doi] 